\title{
Progress in Ultra-High Energy Resolution EELS
}

\author{
TC Lovejoy ${ }^{1 *}$, GJ Corbin ${ }^{1}, \mathrm{~N}$ Dellby ${ }^{1}$, N Johnson ${ }^{1}$, MV Hoffman ${ }^{1}$, A Mittelberger ${ }^{1}$ and OL Krivanek ${ }^{1,2}$ \\ 1. Nion R\&D, Kirkland, WA, USA. \\ 2. Department of Physics, Arizona State University, Tempe AZ, USA. \\ * Corresponding author: lovejoy@nion.com
}

Vibrational spectroscopy in the electron microscope [1], which was made possible by combining stable monochromators with ultra-stable electron energy loss (EEL) spectrometers, continues to progress rapidly. The Nion Iris spectrometer [2], incorporated into the Nion High Energy Resolution Monochromated EELS-STEM (HERMES ${ }^{\mathrm{TM}}$ ) system [3] has led to an EELS era in which $5 \mathrm{meV}$ energy resolution can be attained at $30 \mathrm{keV}$ primary energy, and combined with atom-sized electron probes. The progress in energy resolution is illustrated in Fig. 1, which compares a recent spectrum of guanine (EELS 1) with one published in 2016 (EELS 2), and an FTIR spectrum of the same sample [4]. Reduced tails of the zero loss peak and better energy resolution make the information content of the more recent spectrum resemble the FTIR spectrum more than the older EELS spectrum, especially in the spectrum region between 50 and $220 \mathrm{meV}$.

The energy resolution is now sufficient for identifying energy shifts due to the substitution of ${ }^{13} \mathrm{C}$ for ${ }^{12} \mathrm{C}$ at specific locations in biological molecules. Isotopic sensitivity was recently used to detect and map Lalanine with ${ }^{13} \mathrm{C}$ isotopic substitution at a specific carbon site [6]. Mapping isotopically modified molecules should provide a powerful tool for tracing metabolic pathways in biological samples at $\sim 30 \mathrm{~nm}$ spatial resolution. The $30 \mathrm{~nm}$ limit arises because vibrational spectra from biological samples are best acquired in an aloof geometry, i.e. with the electron beam in the vacuum outside the sample. This selects long-range dipole scattering events and decreases the potentially available spatial resolution, but comes with a significant advantage: it largely avoids radiation damage [4].

Much better spatial resolution can be achieved when the scattering angles accepted into the spectrometer are large, and when small-angle dipole scattering events are excluded from the EELS by setting up "darkfield EELS" collection geometry. This method has been shown to reach nm-level spatial resolution in vibrational mapping in $\mathrm{BN}$ [7], and recently even 2 Å-level spatial resolution [8]. In non-polar materials such as $\mathrm{Si}$, dipole scattering is not dominant, and the high-scattering angle signal can be used to achieve 2 A spatial resolution in phonon maps without using the dark-field EELS geometry, by simply making sure that large scattering angles contribute to the phonon map [9].

Another rich lode of information lies in exploring the angular dependence of phonon scattering, as recently shown by Hage et al. [10], who measured vibrational losses $(\omega)$ as a function of scattering angle and hence momentum transfer $(q)$ to create dispersion plots $\omega$ vs $q$ along high symmetry directions in the Brillouin zone (BZ). The phonon signal at finite angles, however, can be quite weak, and collection efficiency therefore becomes a major concern. Hage et al. collected the information along the momentum axis serially, by using a small EELS collection aperture to record spectra for different momenta (q vectors). With collection times of several minutes per q point and many q points per direction in the BZ, the acquisition took tens of minutes to hours for a complete $\omega$-q data set. 
In Fig. 2 we explore parallel acquisition of $\omega$-q data sets. We use a slot aperture in the spectrometer entrance plane (125 um x $2 \mathrm{~mm}$ ) and rotate the diffraction pattern by adjusting the microscope's projector lenses so that selected high-symmetry directions in the Brillouin zone are sampled by the slot. Parallel acquisition improves the collection efficiency and reduces the effect of system variables that can change over time and limit quantitative analysis. The figure shows an $\omega$-q diagram from hexagonal BN, acquired in two parallel acquisitions of 5 minutes each along two high symmetry directions in the hexagonal BZ: $\Gamma-\mathrm{M}-\Gamma$ and $\Gamma-\mathrm{K}-\mathrm{M}-\mathrm{K}-\Gamma$.

References:

[1] OL Krivanek et al., Nature 514 (2014), p. 209; T Miata et al., Microscopy 63 (2014), p. 377.

[2] TC Lovejoy et al., Microsc. Microanal. 24 (S1) (2018), p. 446.

[3] OL Krivanek et al., Microscopy 62 (2013), p. 3.

[4] P Rez et al., Nature Comms 7, p. 10945. doi: 10.1038/ncomms10945

[5] G Radtke et al., these proceedings

[6] J Hachtel et al., Science 363 (2019), p. 525.

[7] C Dwyer et al., Phys. Rev. Lett 117 (2016), p. 256101.

[8] FS Hage et al., Phys. Rev. Lett 122 (2019), p. 016103.

[9] K Venkatraman et al., arXiv:1812.08895 (2018).

[10] FS Hage et al., Sci. Adv. 4 (2018), p. 1. eaar7495.

Figure 1. (right) Vibrational spectra of guanine. EELS 2 and FTIR were recorded in 2015 and published in 2016 [4], EELS 1 was recorded in 2019. (30kV, $5.9 \mathrm{meV}$ resolution, $100 \mathrm{~ms}$ nonsaturated spectra $\mathrm{x} 2,000$, aligned and summed, 50 $\mathrm{nm}$ aloof, $\alpha / 2=\beta / 2=10 \mathrm{mrad}, 1 \mathrm{pA}$.)
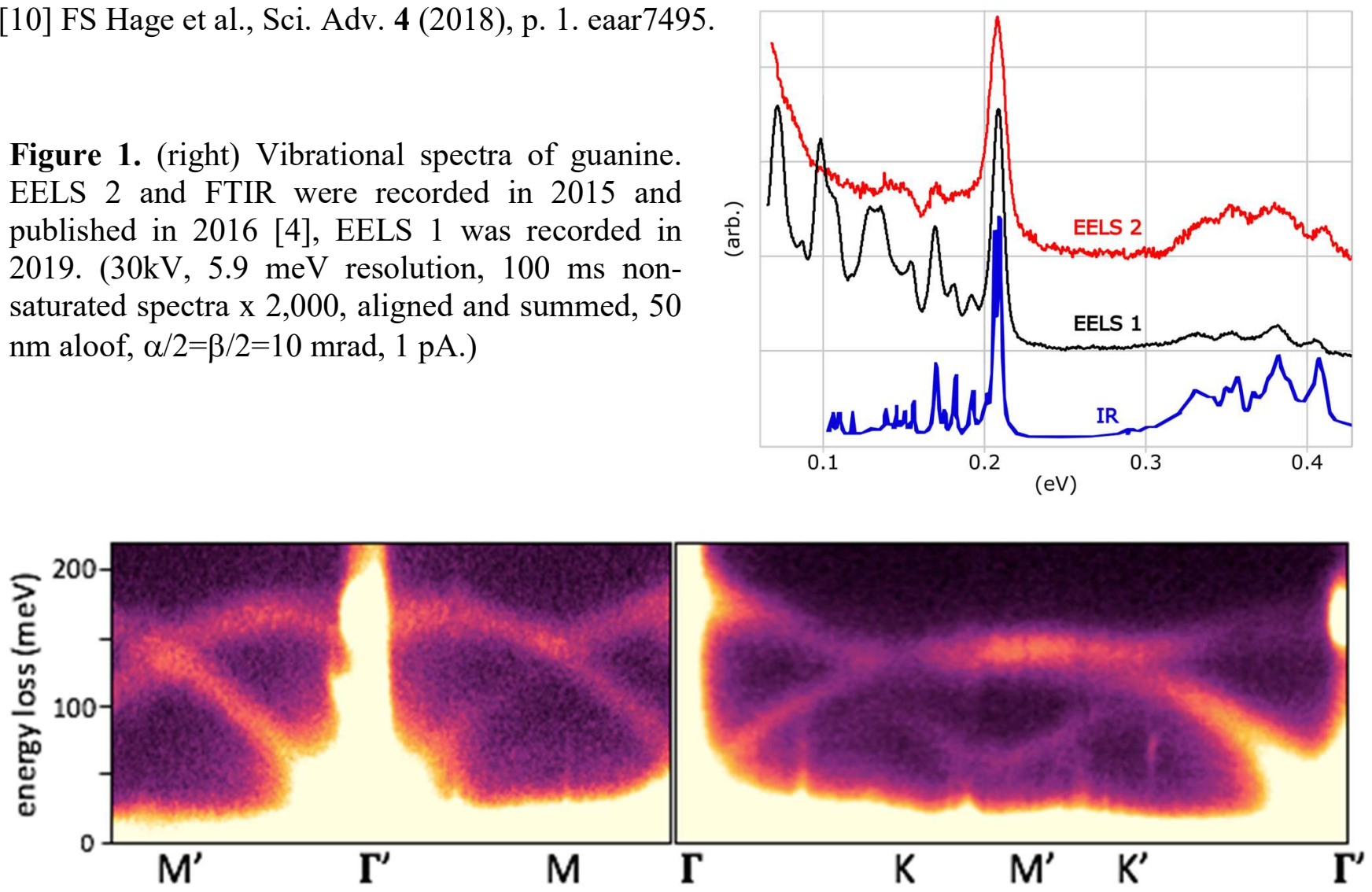

Figure 2. $\omega$-q experimental diagram obtained from hexagonal $\mathrm{BN}$ in two parallel acquisitions (left) spanning $\Gamma-\mathrm{M}-\Gamma^{\prime}-\mathrm{M}^{\prime}$ and (right) $\Gamma-\mathrm{K}-\mathrm{M}^{\prime}-\mathrm{K}^{\prime}-\Gamma^{\prime}$. The curves show clearly the dispersion of the phonon energies as a function of momentum. $30 \mathrm{kV}, 15 \mathrm{pA}, 20 \mathrm{meV}$ resolution, $\alpha / 2=1.5 \mathrm{mrad}$, collection fullangles $\beta_{1} \times \beta_{2}$ approximately $3 \mathrm{mrad} \times 50 \mathrm{mrad}$. 